\title{
Viscous Fluticasone in Eosinophilic Esophagitis: a Suitable Step Forward
}

\author{
Racca $F^{1 *}$, Pellegatta $G^{2}$, Heffler $E^{1,3}$, Repici $A^{2,3}$ and Canonica $G W^{1,3}$ \\ ${ }^{1}$ Personalized Medicine, Asthma and Allergy, IRCCS Humanitas Research Hospital, Rozzano, Italy \\ ${ }^{2}$ Digestive Endoscopy Unit, Department of Gastroenterology, IRCCS Humanitas Research Hospital, Rozzano, Italy \\ ${ }^{3}$ Department of Biomedical Sciences, Humanitas University, Pieve Emanuele, Italy
}

*Corresponding author: Francesca Racca Personalized Medicine, Asthma and Allergy, Humanitas Clinical and Research Center, IRCCS Rozzano, Italy.

To Cite This Article: Racca F, Pellegatta G, Heffler E, Repici A, Canonica G W. Viscous Fluticasone in Eosinophilic Esophagitis: a Suitable Step Forward. Am J Biomed Sci \& Res. 2021 - 13(1). AJBSR.MS.ID.001827. DOI: 10.34297/AJBSR.2021.13.001827.

Received: 䟧 May 17, 2021; Published: 眥 May 27, 2021

\begin{abstract}
Eosinophilic esophagitis (EoE) is a chronic immune mediated disease of the esophagus whose incidence has risen rapidly and is now the first cause of dysphagia in the adult population. Symptoms significantly impact on quality of life and the chronic inflammation, if left untreated, can lead to fibrosis and strictures. Fluticasone propionate (FP) and budesonide (BUD), currently represent one of the first-line therapy for EoE and their efficacy has been demonstrated in multiple studies. However, no current FDA-approved medications exist in the United States, while an orodispersible budesonide tablet was officially approved in Europe but is still unavailable in many Countries. This has led to the routine "off label" use of swallowed metered-dose inhaler with suboptimal delivery. Oral viscous budesonide (OVB) has proved superior to swallowed nebulizer formulation because of a longer drug mucosal contact time. The use of BUD in the form of OVB has gradually surpassed the use of FP. Data on inhaled glucocorticoids, however, have shown that FP has better pharmacological characteristics than BUD resulting in a higher therapeutic index. Comparison between FP and BUD has been established solely using FP nebulizer vs OVB. In clinical trials, FP has been tested only in the form of swallowed spray and oro-dispersible tablets. Only two studies have published data on powder based FP viscous preparations, but liquid-based viscous FP solutions might have a higher potential and clinical data are absent from literature. Viscous suspensions proved to be non-inferior to esophagus-specific targeted budesonide formulations. Until more standardized esophagus-targeted formulations become easily available, there is a need to ameliorate topical steroids formulations with wide availability. Fluticasone viscous solutions have the potential of meeting these needs and would deserve more clinical evidences to support its clinical use. Moreover, possible alternative viscous mediums to sucralose, such as honey or xanthan gum should be considered.
\end{abstract}

Keywords: Eosinophilic Esophagitis; Fluticasone; Budesonide; Oral Viscous Budesonide; Swallowed topical steroids

Abbreviations: EoE: Eosinophilic Esophagitis; STC: Swallowed Topical Corticosteroids; PPI: Proton Pump Inhibitor, FED: Food-Elimination-Diets, FP: Fluticasone Propionate; BUD: Budesonide; MDI: Metered-Dose Inhaler; OVB: Oral Viscous Budesonide

\section{Introduction}

Eosinophilic esophagitis (EoE) is a chronic immune-mediated disease of the esophagus characterized clinically by symptoms related to esophageal dysfunction and histologically by eosinophilpredominant inflammation [1,2]. EoE can affect all age groups, with an incidence peak between the third and the fifth decade of life. Since its first recognition in the '70s, EoE incidence has risen rapidly over the last 15 years both in the US [3] and in Europe [4,5] and is now recognized as the first cause of dysphagia in the adult population [6].
The main clinical manifestation of EoE in adults is dysphagia and food impaction after ingestion of solid foods. In children and infants EoE symptoms might be subtler with failure to thrive, vomiting, nausea, regurgitation, abdominal pain, food aversion and feeding problems [7,8]. Natural history studies revealed that EoE is a chronic disease that significantly impacts on quality of life, including vitality and general health scores [9] and, if left untreated, results in continued inflammation [10] and consequent complications such as fibrosis and strictures [11]. 


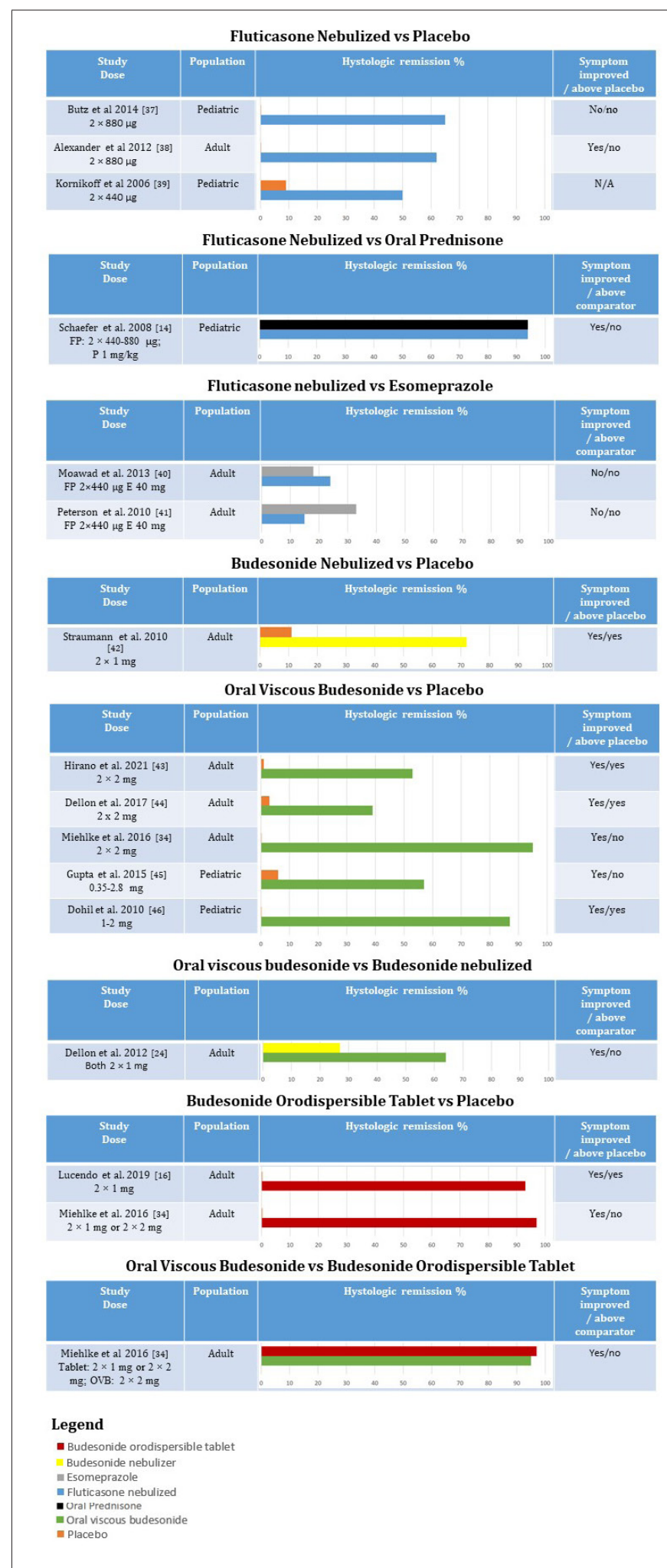

Figure 1: summarizes results from randomized clinical trials assessing efficacy of topical steroids in EoE.

Figure1 Abbreviations: OVB: Oral Viscous Budesonide; FP: Fluticasone propionate; E: esomeprazole; P: Prednisone.
Corticosteroids have been the first treatment for EoE since its recognition as a distinct disease [12]. At first, systemic steroids were employed but subsequent studies demonstrated that topical steroids were able to achieve comparable results, carrying a lower risk of systemic adverse effects $[13,14]$. Swallowed topical corticosteroids (STC) currently represent one of the first-line therapy for EoE, together with proton pump inhibitor (PPI) and food-elimination-diets (FED) [2] and remain today one of the most efficacious therapeutic approach in both children and adults [15]. Multiple studies have demonstrated that STC are able to decrease clinical symptoms, improve histological features of the disease inducing histologic remission in 39\%-100\% of patients [10,16-18], to reverse EoE transcriptome [19] and even decrease fibrosis [20]. Reported adverse effects were mucosal esophageal candidiasis (described in up to $10 \%$ of patients) and rarely adrenal axis suppression, bone demineralization, and diminished growth $[21,22]$.

Fluticasone propionate (FP) and budesonide (BUD) are the most studied and used molecules for EoE treatment in literature (Figure 1). However, no current FDA-approved medications exist for the treatment of EoE in the United States [16,23], while an orodispersible budesonide tablet (Jorveza ${ }^{\circledR}$ ) was officially approved in June 2018 in Europe for EoE induction therapy but is still unavailable in many Countries, such as Italy. This has led to the routine "off label" administration of preparations for bronchial or intranasal delivery, mainly in the form of asthma metered-dose inhaler (MDI) administered to the esophagus by swallowing. The swallowed use of an aerosolized compound can be challenging and can lead to a variable amount of the active drug being deposited into the upper and lower airways and in the ambient air with suboptimal delivery and less than ideal targeting of esophageal mucosa [24]. Moreover, given the high prevalence of concomitant asthma with EoE, patients may habitually continue to inhale the MDI or might follow standard instructions on inhalation techniques provided with the drug.

Thus, a particular budesonide-based preparation, named oral viscous budesonide (OVB), created by mixing liquid budesonide intended for nebulized administration with sucralose or other viscous agents to create a slurry consistency, was developed and has proved superior to the same amount of drug delivered by swallowing a nebulized mist [24] with a longer drug mucosal contact time confirmed scintigraphically. The use of BUD in the form of OVB has, since then, risen and gradually surpassed the use of FP, also for the publication of retrospective and prospective data assessing its superiority in terms of histological and clinical response [25, 26]. 
However, data on inhaled glucocorticoids used in asthma have shown that FP has a higher potency than BUD, with a higher receptor affinity $[27,28]$. Moreover, FP has demonstrated a lower bioavailability than BUD, longer lung retention times and a higher dose to which a $20 \%$ systemic cortisol suppression have been shown, thus resulting in a higher therapeutic index [29]. These properties have not yet been demonstrated in the esophagus, but it feels rational to postulate that FP may behave similarly in EoE therapy, making it the most effective and safe molecule of the pair. Direct comparison between FP and BUD has been established solely using fluticasone nebulizer vs OVB $[25,26]$, and the superiority of OVB on FP could be explained not by its intrinsic anti-inflammatory properties but by the more prolonged contact between the mucosa and the medication due to the sucralose utilized in OVB, just as demonstrated by the previous study comparing BUD suspension and spray formulation [30]. In clinical trials, FP has been tested only in the form of swallowed spray and lately oro-dispersible tablets [31]. Up to date, only two studies, both retrospective, have published data on fluticasone preparations, both powder based, aimed to increase esophageal delivery. Kia et al. [32] proposed an orally administered powder formulation of fluticasone and described a decrease of eosinophilic peak densities to $<15$ eos/ hpf in $75 \%$ of patients, together with improvement in dysphagia symptoms and endoscopic findings of furrows and exudates [32]. Ketchem et al. [33] formulated a viscous suspension by mixing powdered fluticasone with a Methocel gel reporting significant improvement in EoE clinical and endoscopic findings, with trend towards improvement in eosinophil counts. This is notable because the cohort was composed by three-quarters of patients previously non responders to STC (mostly budesonide or fluticasone spray) and/or FED. In particular, 35\% of these patients achieved histological remission ( $<15$ eos/hpf) with compounded fluticasone treatment.

These data are surely of great interest with promising results and in addition the ease of stock and transportation of a powder formula is surely alluring. However, liquid-based viscous FP might have a higher potential for drug delivery even if clinical data are still absent from literature. Viscous suspensions proved to be noninferior to esophagus-specific targeted budesonide formulations (effervescent tablet for orodispersible use) in a European phase-IImulticenter trial with a randomized, double blind, double-dummy, placebo-controlled design, although the effervescent formulation was preferred by patients due to ease of use [34].

Until more standardized esophagus-targeted formulations of topical steroids become commercially available, there is a need to develop and ameliorate the esophageal delivery of topical steroids formulations that could be maximally effective and safe. Moreover, possible alternative viscous medium to sucralose, a non-absorbable sugar with potential of gastrointestinal symptoms at high doses, should be considered. For example, some reports exist on the efficacy of honey- and xanthan gum-based solutions. In particular, these articles reported comparable mucosal contact time and higher palatability of honey based solutions $[35,36]$ and a higher mucosal contact time with comparable palatability of xanthan gum-based formula [36] compared to sucralose-based formula. Based on the clinical and pharmacological evidence reported above, we believe that fluticasone viscous solutions have the potential of meeting the mentioned needs and would deserve more clinical evidences to support and finalize its clinical use [37-46].

\section{Conflict of Interest}

The Authors do not declare any conflict of interest

\section{References}

1. Frederic Clayton, Kathryn Peterson (2018) Eosinophilic Esophagitis: Pathophysiology and Definition. Gastrointest Endosc Clin N Am 28(1): $1-14$.

2. Alfredo J Lucendo, Javier Molina Infante, Angel Arias, Ulrike von Arnim, Albert J Bredenoord, et al. (2017) Guidelines on eosinophilic esophagitis: evidence-based statements and recommendations for diagnosis and management in children and adults. United European Gastroenterol J 5(3): 335-358.

3. Ganapathy A Prasad, Jeffery A Alexander, Cathy D Schleck, Alan R Zinsmeister, Thomas C Smyrk, et al. (2009) Epidemiology of eosinophilic esophagitis over three decades in Olmsted County, Minnesota. Clin Gastroenterol Hepatol 7(10): 1055-1061.

4. Van Rhijn B D, Verheij J, Smout A J, Bredenoord A J (2013) Rapidly increasing incidence of eosinophilic esophagitis in a large cohort. Neurogastroenterol Motil 25(1): 47-52.

5. Petr Hruz, Alex Straumann, Christian Bussmann, Pius Heer, Hans Uwe Simon, et al. (2011) Escalating incidence of eosinophilic esophagitis: a 20-year prospective, population-based study in Olten County, Switzerland. J Allergy Clin Immunol. 128(6): 1349-1350.

6. F J Moawad, A M Schoepfer, E Safroneeva, M R Ally, Y J Chen, et al. (2014) Eosinophilic oesophagitis and proton pump inhibitor-responsive oesophageal eosinophilia have similar clinical, endoscopic and histological findings. Aliment Pharmacol Ther 39(6): 603-608.

7. Pooja Mehta, Glenn T Furuta (2015) Eosinophils in Gastrointestinal Disorders: Eosinophilic Gastrointestinal Diseases, Celiac Disease, Inflammatory Bowel Diseases, and Parasitic Infections. Immunol Allergy Clin North Am. 35(3): 413-437.

8. Richard J Noel, Philip E Putnam, Marc E Rothenberg (2004) Eosinophilic esophagitis. N Engl J Med 351(9): 940-941.

9. Bram D van Rhijn, Pim W Weijenborg, Joanne Verheij, Marius A van den Bergh Weerman, Caroline Verseijden, et al. (2014) Proton pump inhibitors partially restore mucosal integrity in patients with proton pump inhibitor-responsive esophageal eosinophilia but not eosinophilic esophagitis. Clin Gastroenterol Hepatol 12(11): 1815-1823.

10. Evan S Dellon, Chris A Liacouras, Javier Molina Infante, Glenn T Furuta, Jonathan M Spergel, Et al. (2018) Updated International Consensus Diagnostic Criteria for Eosinophilic Esophagitis: Proceedings of the AGREE Conference. Gastroenterology155(4):1022-1033.

11. Schoepfer A M, Safroneeva E, Bussmann C, Kuchen T, Portmann S, et al. (2013) Delay in diagnosis of eosinophilic esophagitis increases risk for stricture formation in a time-dependent manner. Gastroenterology 145(6): 1230-1236. 
12. C A Liacouras, W J Wenner, K Brown, E Ruchelli (1998) Primary eosinophilic esophagitis in children: successful treatment with oral corticosteroids. J Pediatr Gastroenterol Nutr 26(4): 380-385.

13. W A Faubion Jr, J Perrault, L J Burgart, N N Zein, M Clawson, et al. (1998) Treatment of eosinophilic esophagitis with inhaled corticosteroids. Pediatr Gastroenterol Nutr 27(1): 90-93.

14. Elizabeth $\mathrm{T}$ Schaefer, Joseph F Fitzgerald, Jean P Molleston, Joseph M Croffie, Marian D Pfefferkorn, et al. (2008) Comparison of oral prednisone and topical fluticasone in the treatment of eosinophilic esophagitis: a randomized trial in children. Clin Gastroenterol Hepatol 6(2): 165-173.

15. Theodore Rokkas, Yaron Niv, Peter Malfertheiner (2021) A Network Meta-Analysis of Randomized Controlled Trials on the Treatment of Eosinophilic Esophagitis in Adults and Children. J clin gastroenterol 55(5): 400-410.

16. Alfredo J Lucendo, Stephan Miehlke, Christoph Schlag, Michael Vieth, Ulrike von Arnim, et al. (2019) Efficacy of Budesonide Orodispersible Tablets as Induction Therapy for Eosinophilic Esophagitis in a Randomized Placebo-Controlled Trial. Gastroenterology 157(1): 74-86.

17. Cary C Cotton, Swathi Eluri, W Asher Wolf, Evan S Dellon (2017) SixFood Elimination Diet and Topical Steroids are Effective for Eosinophilic Esophagitis: A Meta-Regression. Dig Dis Sci 62(9): 2408-2420.

18. Li Xiao Hao, Yi Lu, Tao Li, Biao Gong (2021) A meta-analysis of efficacy of topical steroids in eosinophilic esophagitis: From the perspective of histologic, clinical, and endoscopic outcome. Gastroenterol Hepatol. 44(4): 251-260.

19. Carine Blanchard, Melissa K Mingler, Maria Vicario, J Pablo Abonia, Yi Ying Wu, et al. (2007) IL-13 involvement in eosinophilic esophagitis: transcriptome analysis and reversibility with glucocorticoids. J Allergy Clin Immunol 120(6): 1292-1300.

20. S S Aceves, R O Newbury, D Chen, J Mueller, R Dohil, H Hoffman, et al. (2010) Resolution of remodeling in eosinophilic esophagitis correlates with epithelial response to topical corticosteroids. Allergy 65(1): 109116.

21. Glenn T Furuta, David A Katzka (2015) Eosinophilic Esophagitis. N Engl J Med 373(17): 1640-1648.

22. Doerthe A Andreae, Matthew G Hanna, Margret S Magid, Stefano Malerba, Michael H Andreae, et al. (2016) Swallowed Fluticasone Propionate Is an Effective Long-Term Maintenance Therapy for Children with Eosinophilic Esophagitis. Am J Gastroenterol 111(8): 1187-1197.

23. Ikuo Hirano, Stuart Spechler, Glenn Furuta, Evan S Dellon (2017) White Paper AGA: Drug Development for Eosinophilic Esophagitis. Clin Gastroenterol Hepatol 15(8): 1173-1183.

24. Evan S Dellon 1, Arif Sheikh, Olga Speck, Kimberly Woodward, Ann B Whitlow, et al (2012) Viscous topical is more effective than nebulized steroid therapy for patients with eosinophilic esophagitis. Gastroenterology 143(2): 321-324.

25. Dustin Albert, Theresa A Heifert, Steve B Min, Corinne L Maydonovitch, Thomas P Baker, et al. (2016) Comparisons of Fluticasone to Budesonide in the Treatment of Eosinophilic Esophagitis. Dig Dis Sci 61(7): 19962001.

26. Evan S Dellon, John T Woosley, Ashley Arrington, Sarah J McGee, Jacquelyn Covington, et al (2019) Efficacy of Budesonide vs Fluticasone for Initial Treatment of Eosinophilic Esophagitis in a Randomized Controlled Trial. Gastroenterology 157(1): 65-73.

27. Melanie Hubner , Gunther Hochhaus, Hartmut Derendorf (2005) Comparative pharmacology, bioavailability, pharmacokinetics, and pharmacodynamics of inhaled glucocorticosteroids. Immunol Allergy Clin North Am 25(3): 469-488.
28. L P Nielsen, R Dahl (2000) Therapeutic ratio of inhaled corticosteroids in adult asthma. A dose-range comparison between fluticasone propionate and budesonide, measuring their effect on bronchial hyperresponsiveness and adrenal cortex function. Am J Respir Crit Care Med 162(6): 2053-2057.

29. Daley Yates P T (2015). Inhaled corticosteroids: potency, dose equivalence and therapeutic index. Br J Clin Pharmacol 80(3): 372-380.

30. Molina Infante J, Lucendo A J (2015) Update on topical steroid therapy for eosinophilic esophagitis. Gastroenterol Hepatol 38(6): 388-397.

31. Simon Nennstiel, Christoph Schlag (2020) Treatment of eosinophlic esophagitis with swallowed topical corticosteroids. World J Gastroenterol 26(36): 5395-5407.

32. L Kia, M Nelson, A Zalewski, D Gregory, N Gonsalves, et al. (2018) Oral delivery of fluticasone powder improves esophageal eosinophilic inflammation and symptoms in adults with eosinophilic esophagitis. Dis Esophagus 31:(12).

33. Corey J Ketchem, Craig C Reed, Zoe Stefanadis, Evan S Dellon (2020) Treatment with compounded fluticasone suspension improves the clinical, endoscopic, and histologic features of eosinophilic esophagitis. Dis Esophagus. doaa120.

34. Stephan Miehlke, Petr Hruz, Michael Vieth, Christian Bussmann, Ulrike von Arnim, et al. (2016) A randomised, double-blind trial comparing budesonide formulations and dosages for short-term treatment of eosinophilic oesophagitis. Gut 65(3): 390-399.

35. Juhee Lee, Michelle Shuker, Terri Brown-Whitehorn, Antonella Cianferoni, Laura Gober, et al. (2016) Oral viscous budesonide can be successfully delivered through a variety of vehicles to treat eosinophilic esophagitis in children. J Allergy Clin Immunol Pract 4(4): 767-768.

36. Jody N Hefner, Robin S Howard, Robert Massey, Miland Valencia, Derek J Stocker et al. (2016) A Randomized Controlled Comparison of Esophageal Clearance Times of Oral Budesonide Preparations. Dig Dis Sci 61(6): 1582-1590

37. Bridget K Butz, Ting Wen, Gerald J Gleich, Glenn T Furuta, Jonathan Spergel, et al. (2014) Efficacy, dose reduction, and resistance to high-dose fluticasone in patients with eosinophilic esophagitis. Gastroenterology 147(2): 324-33.

38. Jeffrey A Alexander, Kee Wook Jung, Amindra S Arora, Felicity Enders, David A Katzka, et al. (2012) Swallowed fluticasone improves histologic but not symptomatic response of adults with eosinophilic esophagitis. Clin Gastroenterol Hepatol 10(7): 742-749.

39. Michael R Konikoff, Richard J Noel, Carine Blanchard, Cassie Kirby, Sean C Jameson, et al. (2006) A randomized, double-blind, placebo-controlled trial of fluticasone propionate for pediatric eosinophilic esophagitis. Gastroenterology 131(5): 1381-1391.

40. Fouad J Moawad, Ganesh R Veerappan, Johnny A Dias, Thomas P Baker Corinne L Maydonovitch, et al. (2013). Randomized controlled trial comparing aerosolized swallowed fluticasone to esomeprazole for esophageal eosinophilia. Am J Gastroenterol 108(3): 366-372.

41. Kathryn A Peterson, Kristen L Thomas, Kristen Hilden, Lyska L Emerson, Jason C Wills, et al. (2010). Comparison of esomeprazole to aerosolized, swallowed fluticasone for eosinophilic esophagitis. Dig Dis Sci 55(5): 1313-1319.

42. Alex Straumann, Sebastien Conus, Lukas Degen, Stephanie Felder, Mirjam Kummer, et al (2010). Budesonide is effective in adolescent and adult patients with active eosinophilic esophagitis. Gastroenterology 139(5): 1526-1537.

43. Ikuo Hirano, Margaret H Collins, David A Katzka, Vincent A Mukkada, Gary W Falk, et al. (2021) Budesonide Oral Suspension Improves Outcomes in Patients With Eosinophilic Esophagitis: Results from a Phase 3 Trial. Clin Gastroenterol Hepatol. 1542-3565(21): 00456-0. 
44. Evan S Dellon , David A Katzka, Margaret H Collins, Mohamed Hamdani, Sandeep K Gupta, et al. (2017) Budesonide Oral Suspension Improves Symptomatic, Endoscopic, and Histologic Parameters Compared With Placebo in Patients With Eosinophilic Esophagitis. Gastroenterology 152(4): 776-786

45. Sandeep K Gupta, Joanne M Vitanza, Margaret H Collins (2015) Efficacy and safety of oral budesonide suspension in pediatric patients with eosinophilic esophagitis. Clin Gastroenterol Hepatol 13(1): 66-76.
46. Ranjan Dohil, Robert Newbury, Lyman Fox, John Bastian, Seema Aceves (2010) Oral viscous budesonide is effective in children with eosinophilic esophagitis in a randomized, placebo-controlled trial. Gastroenterology 139(2): 418-429. 